\title{
ON IMAGE FORMULAE LEADING TO FRACTIONAL KINETIC EQUATIONS SOLUTIONS VIA SUMUDU GENERALIZED K-BESSEL FUNCTIONS
}

\author{
KOTTAKKARAN S. NISAR ${ }^{1}$, MOHEB S. ABOUZAID ${ }^{2,3}$, FETHI BIN M. BELGACEM $^{4}$
}

ABSTRACT. Recently, representation formulae and monotonicity properties of generalized k-Bessel functions, Wk v,c., were established and studied by SR Mondal [24]. In this paper, we pursue and investigate some of their image formulae. We then extract solutions for fractional kinetic equations, involving Wk $\mathrm{v}, \mathrm{c}$, by means of their Sumudu transforms. In the process, Important special cases are then revealed, and analyzed.

\section{INTRODUCTION}

The k-Bessel function of the first kind defined by the following series [30] (also, see [10]):

$$
J_{k, \xi}^{\eta, \delta}(z):=\sum_{n=0}^{\infty} \frac{(\eta)_{n, k}}{\Gamma_{k}(\delta n+\xi+1)} \frac{(-1)^{n}(z / 2)^{n}}{(n !)^{2}},
$$

where $k \in \mathbb{R} ; \xi, \eta, \delta, \in \mathbb{C} ; \Re(\delta)>0$ and $\Re(\xi)>0$.

Here $(\eta)_{n, k}$ is the $k$-Pochhammer symbol defined by (see [13])

$$
(\eta)_{n, k}=\left\{\begin{array}{c}
\frac{\Gamma_{k}(\eta+n k)}{\Gamma_{k}(\eta)} \quad(k \in \mathbb{R} ; \eta \in \mathbb{C} \backslash\{0\}) \\
\eta(\eta+k) \ldots(\eta+(n-1) k) \quad(n \in \mathbb{N} ; \eta \in \mathbb{C})
\end{array}\right.
$$

while $\Gamma_{k}(z)$ denotes the $k$-gamma function defined by (see [13])

$$
\Gamma_{k}(z)=\int_{0}^{\infty} e^{-\frac{t^{k}}{k}} t^{z-1} d t, \quad \Re(z)>0, \quad k>0 .
$$

For $k=1, \Gamma_{k}(z)$ reduces to $\Gamma(z)$ and have the following relations,

$$
\Gamma_{k}(x)=k^{\frac{x}{k}-1} \Gamma\left(\frac{x}{k}\right)
$$

and

$$
\Gamma_{k}(x+k)=x \Gamma_{k}(x) .
$$

The well known Beta function [29] defined by

$$
\mathfrak{B}(a, b)=\int_{0}^{1} t^{a-1}(1-t)^{b-1} d t=\frac{\Gamma(a) \Gamma(b)}{\Gamma(a+b)}, \quad(a, b>0) .
$$

The generalized hypergeometric function ${ }_{p} F_{q}\left(a_{1}, \ldots, a_{p} ; c_{1}, \ldots, c_{q} ; x\right)$, is given by the power series [29]

$$
{ }_{p} F_{q}\left(a_{1}, \ldots, a_{p} ; c_{1}, \ldots, c_{q} ; z\right)=\sum_{k=0}^{\infty} \frac{\left(a_{1}\right)_{k} \cdots\left(a_{p}\right)_{k}}{\left(c_{1}\right)_{k} \cdots\left(c_{q}\right)_{k}(1)_{k}} z^{k}, \quad|z|<1,
$$

2010 Mathematics Subject Classification. 33C10, 33B15, 34B30, 26A33, 44A20, 33E12.

Key words and phrases. fractional calculus; k-bessel function; fractional kinetic equations; laplace transforms. 
where $c_{i},(i=1,2 . \cdots, q)$ can not be zero or a negative integer. Here $p$ or $q$ or both are allowed to be zero. The series (1.7) is absolutely convergent for all finite $z$ if $p \leq q$ and for $|z|<1$ if $p=q+1$. When $p>q+1$, then the series diverge for $z \neq 0$ and the series does not terminate.

The generalized Wright hypergeometric function ${ }_{p} \psi_{q}(z)$ is given by the series [42]

$$
{ }_{p} \psi_{q}(z)={ }_{p} \psi_{q}\left[\begin{array}{c}
\left(a_{i}, \gamma\right)_{1, p} \\
\left(b_{j}, \eta_{j}\right)_{1, q}
\end{array} \mid z\right]=\sum_{k=0}^{\infty} \frac{\prod_{i=1}^{p} \Gamma\left(a_{i}+\gamma_{i} k\right)}{\prod_{j=1}^{q} \Gamma\left(b_{j}+\eta_{j} k\right)} \frac{z^{k}}{k !},
$$

where $a_{i}, b_{j} \in \mathbb{C}$, and real $\gamma_{i}, \eta_{j} \in \mathbb{R}(i=1,2, \ldots, p ; j=1,2, \ldots, q)$. The asymptotic behavior of this function for large values of argument of $z \in \mathbb{C}$ were studied in $[15,21]$ and under the condition

$$
\sum_{j=1}^{q} \eta_{j}-\sum_{i=1}^{p} \gamma_{i}>-1
$$

The more properties of the Wright function are investigated in [20-22,42,43]. The Mittag-Leffler function $E_{\rho}(z)$ (see, [23]) and $E_{\rho, \eta}(x)$ (see, [41]) respectively defined by

$$
\begin{gathered}
E_{\rho}(z)=\sum_{n=0}^{\infty} \frac{z^{n}}{\Gamma(\rho n+1)},(z, \rho, \in \mathbb{C} ;|z|<0, \Re(\rho)>0) \\
E_{\rho, \eta}(z)=\sum_{n=0}^{\infty} \frac{z^{n}}{\Gamma(\rho n+\eta)},(z, \rho, \eta \in \mathbb{C} ;|z|<0, \Re(\rho)>0, \Re(\eta)>0) .
\end{gathered}
$$

Recently, SR Mondal [24] gives the new generalization of $\mathrm{k}$-Bessel function $\mathrm{W}_{\nu, c}^{\mathrm{k}}$ and is defined by

$$
\mathrm{W}_{\nu, c}^{\mathrm{k}}(x):=\sum_{r=0}^{\infty} \frac{(-c)^{r}}{\Gamma_{\mathrm{k}}(r \mathrm{k}+\nu+\mathrm{k}) r !}\left(\frac{x}{2}\right)^{2 r+\frac{\nu}{\mathrm{k}}},
$$

where $\mathrm{k}>0, \nu>-1$ and $c \in \mathbb{R}$.

The Sumudu transform introduced by Watugala (see $[39,40])$. For more details about Sumudu transform, see ( $[1-9])$. The Sumudu transform over the set functions

is defined by

$$
A=\left\{f(t)\left|\exists M, \tau_{1}, \tau_{2}>0,\right| f(t) \mid<M e^{|t| / \tau_{j}}, \text { if } t \in(-1)^{j} \times[0, \infty)\right\},
$$

$$
G(u)=S[f(t) ; u]=\int_{0}^{\infty} f(u t) e^{-t} d t, u \in\left(-\tau_{1}, \tau_{2}\right) .
$$

The Sumudu transform of $\mathrm{k}-$ Bessel function is given by

$$
\begin{aligned}
S\left[\mathrm{~W}_{\mu, c}^{\mathrm{k}}(x)\right] & =\int_{0}^{\infty} e^{-t} \mathrm{~W}_{\mu, c}^{\mathrm{k}}(u t) d t \\
& =\int_{0}^{\infty} e^{-t} \sum_{r=0}^{\infty} \frac{(-c)^{r}}{\Gamma_{\mathrm{k}}(r \mathrm{k}+\mu+\mathrm{k}) r !}\left(\frac{u t}{2}\right)^{2 r+\frac{\mu}{\mathrm{k}}} d t \\
& =\sum_{r=0}^{\infty} \frac{(-c)^{r}}{\Gamma_{\mathrm{k}}(r \mathrm{k}+\mu+\mathrm{k}) r !} \int_{0}^{\infty} e^{-t}\left(\frac{u t}{2}\right)^{\frac{\mu}{\mathrm{k}}+2 r} d t \\
& =\sum_{r=0}^{\infty} \frac{(-c)^{r} \Gamma\left(2 r+\frac{\mu}{\mathrm{k}}+1\right)}{\Gamma_{\mathrm{k}}(r \mathrm{k}+\mu+\mathrm{k}) r !}\left(\frac{u}{2}\right)^{\frac{\mu}{\mathrm{k}}+2 r},
\end{aligned}
$$

Now, using the relation

$$
\Gamma_{\mathrm{k}}(\gamma)=\mathrm{k}^{\frac{\gamma}{\mathrm{k}}-1} \Gamma\left(\frac{\gamma}{\mathrm{k}}\right)
$$


we have the following

$$
S\left[\mathrm{~W}_{\mu, c}^{\mathrm{k}}(x)\right]=\sum_{r=0}^{\infty} \frac{(-c)^{r} \Gamma\left(\frac{\mu}{\mathrm{k}}+2 r+1\right)}{\mathrm{k}^{r+\frac{\mu}{\mathrm{k}}} \Gamma\left(r+\frac{\mu}{\mathrm{k}}+1\right) r !}\left(\frac{u}{2}\right)^{\frac{\mu}{\mathrm{k}}+2 r} .
$$

Denoting the left hand side by $G(u)$, we have

$$
\begin{aligned}
G(u) & =S\left[\mathrm{~W}_{\mu, c}^{\mathrm{k}}(t) ; u\right] \\
& =\left(\frac{u}{2}\right)^{\frac{\mu}{\mathrm{k}}} k^{-\frac{\mu}{\mathrm{k}}} 1_{1}\left[\begin{array}{c}
\left(\frac{\mu}{\mathrm{k}}+1,2\right) \\
\left(\frac{\mu}{\mathrm{k}}+1,1\right)
\end{array} \mid-\frac{c u^{2}}{4 \mathrm{k}}\right] .
\end{aligned}
$$

In this paper, our aim is to investigate fractional integration of (1.12) including image formulas and solutions of fractional kinetic equation via Sumudu transform.

\section{IMAGE FORMULA OF $W_{v, c}^{\mathrm{k}}(z)$}

The fractional integrals of a function $f(z)$ of order $\eta[32]$ are given by

$$
\left(I_{0+}^{\eta} f\right)(z)=\frac{1}{\Gamma(\eta)} \int_{0}^{z} \frac{f(t)}{(z-t)^{1-\eta}} d t \quad(z>0)
$$

and

$$
\left(I_{-}^{\eta} f\right)(z)=\frac{1}{\Gamma(\eta)} \int_{z}^{\infty} \frac{f(t)}{(t-z)^{1-\eta}} d t \quad(z>0)
$$

The fractional derivatives of a function $f(z)$ of order $\eta[32]$ are given by

$$
\begin{aligned}
\left(D_{0+}^{\eta} f\right)(z) & =\left(\frac{d}{d z}\right)^{[\Re(\eta)]+1}\left(I_{0+}^{1-\eta+[\Re(\eta)]} f\right)(z) \\
& =\frac{1}{1-\eta+[\Re(\eta)]}\left(\frac{d}{d z}\right)^{[\Re(\eta)]+1} \int_{0}^{z} \frac{f(t)}{(z-t)^{\eta-[\Re(\eta)]}} d t \quad(z>0)
\end{aligned}
$$

and

$$
\begin{aligned}
\left(D_{-}^{\eta} f\right)(z) & =\left(\frac{d}{d z}\right)^{[\Re(\eta)]+1}\left(I_{0+}^{1-\eta+[\Re(\eta)]} f\right)(z) \\
& =\frac{1}{1-\eta+[\Re(\eta)]}\left(-\frac{d}{d z}\right)^{[\Re(\eta)]+1} \int_{z}^{\infty} \frac{f(t)}{(t-z)^{\eta-[\Re(\eta)]}} d t \quad(z>0)
\end{aligned}
$$

Now, we give some image formulas of (1.12) using (2.1)-(2.4).

Theorem 1. Let $\mathrm{k}>0, v>-1, \Re(\eta)>0$ and $a, c \in \mathbb{R}$ then

$$
\left(I_{0+}^{\eta} t^{\frac{v}{\mathrm{k}}} W_{v, c}^{\mathrm{k}}(a \sqrt{t})\right)(x)=\mathrm{k}^{\eta}\left(\frac{a}{2}\right)^{-\eta} x^{\frac{v}{\mathrm{k}}+\frac{\eta}{2}} W_{v+\eta \mathrm{k}, c}^{\mathrm{k}}(a \sqrt{x}), \quad(x>0)
$$

Proof. Let the left hand side (LHS) of (2.5) is denoted by $\mathfrak{L}_{\perp}$ and using the (1.12), we get

$$
\mathfrak{L}_{1}=\left(I_{0+}^{\eta} t^{\frac{v}{\mathrm{k}}} W_{v, c}^{\mathrm{k}}(a \sqrt{t})\right)(x)=\left(I_{0+}^{\eta} t^{\frac{v}{\mathrm{k}}} \sum_{r=0}^{\infty} \frac{(-c)^{r}}{\Gamma_{\mathrm{k}}(r \mathrm{k}+v+\mathrm{k}) r !}\left(\frac{a \sqrt{t}}{2}\right)^{2 r+\frac{v}{\mathrm{k}}}\right)(x),
$$

Using (2.1), we have

$$
\mathfrak{L}_{1}=\frac{1}{\Gamma(\eta)} \int_{0}^{x} \frac{t^{\frac{v}{\mathrm{k}}}}{(x-t)^{1-\eta}} \sum_{r=0}^{\infty} \frac{(-c)^{r}}{\Gamma_{\mathrm{k}}(r \mathrm{k}+v+\mathrm{k}) r !}\left(\frac{a \sqrt{t}}{2}\right)^{2 r+\frac{v}{\mathrm{k}}} d t .
$$


Interchanging the summation and integration and then evaluating the inner integral by substituting $t=x u$, we get

$$
\mathfrak{L}_{\perp}=\frac{1}{\Gamma(\eta)} \sum_{r=0}^{\infty} \frac{(-c)^{r}}{\Gamma_{\mathrm{k}}(r \mathrm{k}+v+\mathrm{k}) r !}\left(\frac{a}{2}\right)^{2 r+\frac{v}{\mathrm{k}}} \int_{0}^{1} u^{r+\frac{v}{\mathrm{k}}}(1-u)^{\eta-1} d u
$$

In view of (1.4) and (1.6) we arrived the required result.

Corollary 2.1. If we set $c=1$ in Theorem 1 , then we get the fractional integration of $k$-Bessel function $J_{v}^{k}(x)$ as,

$$
\left(I_{0+}^{\eta} t^{\frac{v}{\mathrm{k}}} J_{v}^{\mathrm{k}}(a \sqrt{t})\right)(x)=\mathrm{k}^{\eta}\left(\frac{a}{2}\right)^{-\alpha} x^{\frac{v}{\mathrm{k}}+\frac{\alpha}{2}} J_{v+\eta \mathrm{k}}^{\mathrm{k}}(a \sqrt{x}) .
$$

which is equation (12) of [16].

Theorem 2. Let $\mathrm{k}>0, v>-1, \Re(\eta)>0$ and $a, c \in \mathbb{R}$ then

$$
\left(I_{-}^{\eta} t^{-\frac{v}{\mathrm{k}}-\eta-1} W_{v, c}^{\mathrm{k}}\left(\frac{a}{\sqrt{t}}\right)\right)(x)=\mathrm{k}^{\eta}\left(\frac{a}{2}\right)^{-\eta} x^{-\frac{v}{\mathrm{k}}+\frac{\eta}{2}-1} W_{v+\eta \mathrm{k}, c}^{\mathrm{k}}\left(\frac{a}{\sqrt{x}}\right), \quad(x>0) .
$$

Proof. Let the LHS of $(2.7)$ is denoted by $\mathfrak{L}_{2}$,

$$
\mathfrak{L}_{2}=\left(I_{-}^{\eta} t^{-\frac{v}{\mathrm{k}}-\eta-1} W_{v, c}^{\mathrm{k}}\left(\frac{a}{\sqrt{t}}\right)\right)(x)
$$

Using (2.2) and using the (1.12), we get

$$
\mathfrak{L}_{2}=\frac{1}{\Gamma(\eta)} \int_{x}^{\infty} \frac{t^{-\frac{v}{\mathrm{k}}-\eta-1}}{(t-x)^{1-\eta}} \sum_{r=0}^{\infty} \frac{(-c)^{r}}{\Gamma_{\mathrm{k}}(r \mathrm{k}+v+\mathrm{k}) r !}\left(\frac{a}{2 \sqrt{t}}\right)^{2 r+\frac{v}{\mathrm{k}}} d t
$$

Interchanging the summation and integration and then evaluating the inner integral by substituting $t=\frac{x}{u}$, we get

$$
\mathfrak{L}_{2}=\frac{1}{\Gamma(\eta)} \sum_{r=0}^{\infty} \frac{(-c)^{r}}{\Gamma_{\mathrm{k}}(r \mathrm{k}+v+\mathrm{k}) r !}\left(\frac{a}{2}\right)^{2 r+\frac{v}{\mathrm{k}}} x^{-\frac{v}{k}-r-1} \int_{0}^{1} u^{1+r+\frac{v}{\mathrm{k}}}(1-u)^{\eta-1} d u,
$$

In view of (1.4) and (1.6) we arrived the required result.

Corollary 2.2. If we set $c=1$ in Theorem 2, we get

$$
\left(I_{-}^{\eta} t^{-\frac{v}{\mathrm{k}}-\eta-1} J_{v}^{\mathrm{k}}\left(\frac{a}{\sqrt{t}}\right)\right)(x)=\mathrm{k}^{\eta}\left(\frac{a}{2}\right)^{-\eta} x^{-\frac{v}{\mathrm{k}}+\frac{\eta}{2}-1} J_{v+\eta \mathrm{k}}^{\mathrm{k}}\left(\frac{a}{\sqrt{x}}\right), \quad(x>0) .
$$

which is equation (13) of [16].

In view (2.3) and (2.4), we have the following left and right handed fractional differentiation as follows:

Theorem 3. Let $\mathrm{k}>0, v>-1, \Re(\eta)>0$ and $a, c \in \mathbb{R}$ then

$$
\left(D_{0+}^{\eta}\left[t^{\frac{v}{2 \mathrm{k}}} W_{v, c}^{\mathrm{k}}(a \sqrt{t})\right]\right)(x)=\mathrm{k}^{-\eta}\left(\frac{a}{2}\right)^{\eta} x^{\frac{v}{2 \mathrm{k}}+\frac{\eta}{2}} W_{v-\eta \mathrm{k}, c}^{\mathrm{k}}(a \sqrt{x}), \quad(x>0) .
$$

Proof. Using the definition of (1.12) and (2.3), we can easily find the required result. So the details are omitted.

Theorem 4. Let $\mathrm{k}>0, v>-1, \Re(\eta)>0$ and $a, c \in \mathbb{R}$ then

$$
\left(D_{-}^{\eta}\left[t^{-\frac{v}{\mathrm{k}}-\eta-1} W_{v, c}^{\mathrm{k}}\left(\frac{a}{\sqrt{t}}\right)\right]\right)(x)=\mathrm{k}^{-\eta}\left(\frac{a}{2}\right)^{\eta} x^{-\frac{v}{\mathrm{k}}-\frac{\eta}{2}-1} W_{v-\eta \mathrm{k}, c}^{\mathrm{k}}\left(\frac{a}{\sqrt{x}}\right), \quad(x>0) .
$$


Proof. Using the definition of (1.12) and (2.4), we can easily find the desired result. So the details are omitted.

Corollary 2.3. If we set $c=1$ in Theorem 3, we get

$$
\left(D_{0+}^{\eta}\left[t^{\frac{v}{2 \mathrm{k}}} W_{1, c}^{\mathrm{k}}(a \sqrt{t})\right]\right)(x)=\mathrm{k}^{-\eta}\left(\frac{a}{2}\right)^{\eta} x^{\frac{v}{2 \mathrm{k}}+\frac{\eta}{2}} J_{v-\eta \mathrm{k}}^{\mathrm{k}}(a \sqrt{x}) \quad(x>0) .
$$

which is equation (14) of [16]

Corollary 2.4. If we set $c=1$ in Theorem 4

$$
\left(D_{-}^{\eta}\left[t^{-\frac{v}{\mathrm{k}}-\eta-1} W_{v, 1}^{\mathrm{k}}\left(\frac{a}{\sqrt{t}}\right)\right]\right)(x)=\mathrm{k}^{-\eta}\left(\frac{a}{2}\right)^{\eta} x^{-\frac{v}{\mathrm{k}}-\frac{\eta}{2}-1} J_{v-\eta \mathrm{k}}^{\mathrm{k}}\left(\frac{a}{\sqrt{x}}\right), \quad(x>0) .
$$

which is equation (15) of [16]

\section{Solution of Generalized Fractional Kinetic Equations (GFKE) involving (1.12)}

In this section, we consider (1.12) to obtain the solution of the fractional kinetic equations using Sumudu transform. For more details about GKFE and its solutions, one can refer various paper available in the literature ( $[11,12,17,18,25-28,31,33-36,44])$.

As mentioned in [19],the destruction rate and the production rate as follows,

$$
\frac{d Q}{d t}=-\mathfrak{d}\left(Q_{t}\right)+\mathfrak{p}\left(Q_{t}\right)
$$

where $Q_{t}$ described by $Q_{t}\left(t^{*}\right)=Q\left(t-t^{*}\right), t^{*}>0$.

If spatial fluctuation and inhomogeneities in the quantity $Q(t)$ are neglected, then (3.1) reduced into

$$
\frac{d Q_{i}}{d t}=-c_{i} Q_{i}(t)
$$

which is the number density of species $i$ at time $t=0$ and $c_{i}>0$ is given by the initial condition $Q_{i}(t=0)=Q_{0}$. Now after integrating and decline the index $i,(3.2)$ reduced into

$$
Q(t)-Q_{0}=-c_{0} \times{ }_{0} D_{t}^{-1} Q(t)
$$

where ${ }_{0} D_{t}{ }^{-1}$ is the Riemann-Liouville fractional integral operator.

Haubold and Mathai [19] gives a generalized form of the fractional kinetic equation (3.2) as follows

$$
Q(t)-Q_{0} f(t)=-c^{\nu} D_{t}^{-\nu} Q(t), \Re(\nu)>0,
$$

where

$$
{ }_{0} D_{t}^{-\nu} f(t)=\frac{1}{\Gamma(\nu)} \int_{0}^{t}(t-u)^{\nu-1} f(u) d u, t>0, \Re(\nu)>0 .
$$

The solution of equation (3.4) is true for

$$
Q(t)=Q_{0} \sum_{n=0}^{\infty} \frac{(-1)^{n}}{\Gamma(\nu n+1)}(c t)^{\nu n} .
$$

The use of Laplace transform [37] to (3.4) gives

$$
\begin{aligned}
L[Q(t)] & =Q_{0} \frac{F(p)}{1+c^{\nu} p^{-\nu}} \\
& =Q_{0} \sum_{n=0}^{\infty}(-c)^{n \nu}(p)^{-n \nu} F(p) ; n \in Q_{0},\left|\frac{c}{p}\right|<1,
\end{aligned}
$$


where

$$
F(p)=L f(t)=\int_{0}^{\infty} e^{-p t} f(t) d t, R(p)>0 .
$$

Theorem 5. If $d>0, \nu>0, \mu>-1, t \in \mathbb{C}$ and $c, \mathrm{k} \in \mathbb{R}$ then the solution the equation

$$
Q(t)=Q_{0} \mathrm{~W}_{\mu, c}^{\mathrm{k}}\left(d^{\nu} t^{\nu}\right)-d_{0}^{\nu} D_{t}^{-\nu} Q(t)
$$

is given by the following formula

$$
\begin{aligned}
Q(t)= & Q_{0} \sum_{r=0}^{\infty} \frac{(-c)^{r} \Gamma\left[\nu\left(2 r+\frac{\mu}{\mathrm{k}}\right)+1\right]}{\Gamma_{\mathrm{k}}(r \mathrm{k}+\mu+\mathrm{k}) r !} \frac{1}{t}\left(\frac{d^{\nu} t^{\nu}}{2}\right)^{2 r+\frac{\mu}{\mathrm{k}}} \\
& \times E_{\nu, \nu\left(2 r+\frac{\mu}{\mathrm{k}}\right)}\left(-d^{\nu} t^{\nu}\right) .
\end{aligned}
$$

where $E_{\nu, \nu\left(2 r+\frac{\mu}{\mathrm{k}}\right)}\left(-d^{\nu} t^{\nu}\right)$ is the generalized Mittag-Leffler function [41]

Proof. The Sumudu transform of Riemann-Lioville fractional integral operators is given by

$$
S\left\{{ }_{0} D_{t}^{-\nu} f(t) ; u\right\}=u^{\nu} G(u)
$$

where $G(u)$ is defined in (1.17). Now applying Sumudu transform both sides of (3.9) and applying the definition of $\mathrm{k}$-Bessel function given in (1.12), we have

$$
\begin{aligned}
Q^{*}(u) & =S[Q(t) ; u] \\
& =Q_{0} S\left[\mathrm{~W}_{\mu, c}^{\mathrm{k}}\left(d^{\nu} t^{\nu}\right) ; u\right]-d^{\nu} S\left[{ }_{0} D_{t}^{-\nu} Q(t) ; u\right] \\
& =Q_{0}\left[\int_{0}^{\infty} e^{-t} \sum_{r=0}^{\infty} \frac{(-c)^{r}}{\Gamma_{\mathrm{k}}(r \mathrm{k}+\mu+\mathrm{k}) r !}\left(\frac{d^{\nu}(u t)^{\nu}}{2}\right)^{2 r+\frac{\mu}{\mathrm{k}}} d t\right] \\
& -d^{\nu} u^{\nu} Q^{*}(u),
\end{aligned}
$$

where

$$
S\left\{t^{\mu-1}\right\}=u^{\mu-1} \Gamma(\mu)
$$

By rearranging terms we get,

$$
\begin{aligned}
& Q^{*}(u)+d^{\nu} u^{\nu} Q^{*}(u) \\
& =Q_{0} \sum_{r=0}^{\infty} \frac{(-c)^{r}}{\Gamma_{\mathrm{k}}(r \mathrm{k}+\mu+\mathrm{k}) r !}\left(\frac{d^{\nu}}{2}\right)^{2 r+\frac{\mu}{\mathrm{k}}} \\
& \times \int_{0}^{\infty} e^{-t}(u t)^{\nu\left(2 r+\frac{\mu}{\mathrm{k}}\right)} d t \\
& =Q_{0} \sum_{r=0}^{\infty} \frac{(-c)^{r} \Gamma\left[\nu\left(2 r+\frac{\mu}{k}\right)+1\right]}{\Gamma_{\mathrm{k}}(r \mathrm{k}+\mu+\mathrm{k}) r !}\left(\frac{u^{\nu} d^{\nu}}{2}\right)^{2 r+\frac{\mu}{\mathrm{k}}},
\end{aligned}
$$

Therefore

$$
\begin{aligned}
Q^{*}(u) & =Q_{0} \sum_{r=0}^{\infty} \frac{(-c)^{r} \Gamma\left[\nu\left(2 r+\frac{\mu}{k}\right)+1\right]}{\Gamma_{\mathrm{k}}(r \mathrm{k}+\mu+\mathrm{k}) r !}\left(\frac{d^{\nu}}{2}\right)^{2 r+\frac{\mu}{\mathrm{k}}} \\
& \times\left\{u^{\nu\left(2 r+\frac{\mu}{\mathrm{k}}\right)} \sum_{n=0}^{\infty}\left[-(d u)^{\nu}\right]^{n}\right\}
\end{aligned}
$$


Applying inverse Sumudu transform of (3.14), and by using

$$
S^{-1}\left\{u^{\nu} ; t\right\}=\frac{t^{\nu-1}}{\Gamma(\nu)}, \Re(\nu)>0,
$$

we have

$$
\begin{aligned}
S^{-1}\left\{Q^{*}(u)\right\} & =Q_{0} \sum_{r=0}^{\infty} \frac{(-c)^{r} \Gamma\left[\nu\left(2 r+\frac{\mu}{k}\right)+1\right]}{\Gamma_{\mathrm{k}}(r \mathrm{k}+\mu+\mathrm{k}) r !}\left(\frac{d^{\nu}}{2}\right)^{2 r+\frac{\mu}{\mathrm{k}}} \\
& \times S^{-1}\left\{\sum_{n=0}^{\infty}(-1)^{n}(d)^{\nu n} u^{\nu\left(2 r+\frac{\mu}{\mathrm{k}}+n\right)}\right\}
\end{aligned}
$$

which gives,

$$
\begin{aligned}
Q(t) & =Q_{0} \sum_{r=0}^{\infty} \frac{(-c)^{r} \Gamma\left[\nu\left(2 r+\frac{\mu}{\mathrm{k}}\right)+1\right]}{\Gamma_{\mathrm{k}}(r \mathrm{k}+\mu+\mathrm{k}) r !}\left(\frac{d^{\nu}}{2}\right)^{2 r+\frac{\mu}{\mathrm{k}}} \\
& \times\left\{\sum_{n=0}^{\infty}(-1)^{n}(d)^{\nu n} \frac{t^{\nu\left(2 r+\frac{\mu}{\mathrm{k}}+n\right)-1}}{\Gamma\left[\nu\left(2 r+\frac{\mu}{\mathrm{k}}+n\right)\right]}\right\} \\
& =Q_{0} \sum_{r=0}^{\infty} \frac{(-c)^{r} \Gamma\left[\nu\left(2 r+\frac{\mu}{\mathrm{k}}\right)+1\right]}{\Gamma_{\mathrm{k}}(r \mathrm{k}+\mu+\mathrm{k}) r !} \frac{1}{t}\left(\frac{d^{\nu} t^{\nu}}{2}\right)^{2 r+\frac{\mu}{\mathrm{k}}} \\
& \times\left\{\sum_{n=0}^{\infty}(-1)^{n}(d)^{\nu n} \frac{t^{\nu n}}{\Gamma\left[\nu\left(2 r+\frac{\mu}{\mathrm{k}}+n\right)\right]}\right\} \\
& =Q_{0} \sum_{r=0}^{\infty} \frac{(-c)^{r} \Gamma\left[\nu\left(2 r+\frac{\mu}{\mathrm{k}}\right)+1\right]}{r ! \Gamma_{\mathrm{k}}(r \mathrm{k}+\mu+\mathrm{k})} \frac{1}{t}\left(\frac{d^{\nu} t^{\nu}}{2}\right)^{2 r+\frac{\mu}{\mathrm{k}}} \\
& \times E_{\nu, \nu\left(2 r+\frac{\mu}{\mathrm{k}}\right)}\left(-d^{\nu} t^{\nu}\right) .
\end{aligned}
$$

which is the desired result.

Corollary 3.1. If we put $\mathrm{k}=1$ in (3.10) then we get the solution of involving Bessel function as: If $d>0, \nu>0, \mu>-1, \in \mathbb{C}$ and $c \in \mathbb{R}$ then the equation

$$
Q(t)=Q_{0} \mathrm{~W}_{\mu, c}^{1}\left(d^{\nu} t^{\nu}\right)-d^{\nu}{ }_{0} D_{t}^{-\nu} Q(t)
$$

have the solution:

$$
\begin{aligned}
Q(t) & =Q_{0} \sum_{r=0}^{\infty} \frac{(-c)^{r} \Gamma(\nu(2 r+\mu)+1)}{\Gamma(r+\mu+1) r !} \frac{1}{t}\left(\frac{d^{\nu} t^{\nu}}{2}\right)^{2 r+\mu} \\
& \times E_{\nu, \nu(2 r+\mu)}\left(-d^{\nu} t^{\nu}\right) .
\end{aligned}
$$

Theorem 6. If $\mathfrak{a}>0, d>0, \mu>-1, t \in \mathbb{C}, \mathfrak{a} \neq d$ and $c, \mathrm{k} \in \mathbb{R}$, then the solution of equation

$$
Q(t)=Q_{0} \mathrm{~W}_{\mu, c}^{\mathrm{k}}\left(d^{\nu} t^{\nu}\right)-\mathfrak{a}^{\nu}{ }_{0} D_{t}^{-\nu} Q(t),
$$

is given by

$$
\begin{aligned}
Q(t) & =Q_{0} \sum_{r=0}^{\infty} \frac{(-c)^{r} \Gamma\left[\nu\left(2 r+\frac{\mu}{\mathrm{k}}\right)+1\right]}{\Gamma_{\mathrm{k}}(r \mathrm{k}+\mu+\mathrm{k}) r !} \frac{1}{t}\left(\frac{d^{\nu} t^{\nu}}{2}\right)^{2 r+\frac{\mu}{\mathrm{k}}+1} \\
& \times E_{\nu, \nu\left(2 r+\frac{\mu}{\mathrm{k}}\right)}\left(-\mathfrak{a}^{\nu} t^{\nu}\right)
\end{aligned}
$$


Proof. Theorem 6 can be proved in parallel with the proof of theorem 5 . So the details of proofs are omitted.

Corollary 3.2. By putting $\mathrm{k}=1$ in theorem 6 , we get the solution of fractional kinetic equation involving classical Struve function: If $\mathfrak{a}>0, d>0, \mu>-1, t \in \mathbb{C}, \mathfrak{a} \neq d$, then the equation

$$
Q(t)=Q_{0} \mathrm{~W}_{\mu, c}^{1}\left(d^{\nu} t^{\nu}\right)-\mathfrak{a}^{\nu}{ }_{0} D_{t}^{-\nu} Q(t)
$$

is given by the following formula

$$
\begin{aligned}
Q(t) & =Q_{0} \sum_{r=0}^{\infty} \frac{(-c)^{r} \Gamma[\nu(2 r+\mu+1)]}{\Gamma(r+\mu+1)} \frac{1}{t}\left(\frac{d^{\nu} t^{\nu}}{2}\right)^{2 r+\mu+1} \\
& \times E_{\nu, \nu(2 r+\mu)}\left(-\mathfrak{a}^{\nu} t^{\nu}\right)
\end{aligned}
$$

Theorem 7. If $d>0, \nu>0, \mu>-1, t \in \mathbb{C}$ and $c, \mathrm{k} \in \mathbb{R}$, then the solution of

$$
Q(t)=Q_{0} \mathrm{~W}_{\mu, c}^{\mathrm{k}}\left(t^{\nu}\right)-d^{\nu}{ }_{0} D_{t}^{-\nu} Q(t)
$$

is given by

$$
\begin{aligned}
Q(t) & =N_{0} \sum_{r=0}^{\infty} \frac{(-c)^{r} \Gamma[\nu(2 r+\mathrm{k})+1]}{\Gamma_{\mathrm{k}}(r \mathrm{k}+\mu+\mathrm{k}) r !} \frac{1}{t}\left(\frac{t}{2}\right)^{2 r+\mathrm{k}+1} \\
& \times E_{\nu, \nu\left(2 r+\frac{\mu}{\mathrm{k}}\right)}\left(-d^{\nu} t^{\nu}\right)
\end{aligned}
$$

Proof. The proofs of theorem 7 would run parallel to those of theorem 5 .

Corollary 3.3. If we set $\mathrm{k}=1$ then (3.23) reduced as follows:

If $d>0, \nu>0, \mu>-1, t \in \mathbb{C}$ and $c \in \mathbb{R}$, then the solution of the following equation

$$
Q(t)=Q_{0} \mathrm{~W}_{\mu, c}^{1}\left(t^{\nu}\right)-d^{\nu}{ }_{0} D_{t}^{-\nu} N(t),
$$

is given by the formula

$$
\begin{aligned}
Q(t) & =Q_{0} \sum_{r=0}^{\infty} \frac{(-c)^{r} \Gamma[\nu(2 r+\mu)+1]}{\Gamma(r+\mu+\mathrm{k}) r !} \frac{1}{t}\left(\frac{t}{2}\right)^{2 r+\mu+1} \\
& \times E_{\nu, \nu(2 r+\mu)}\left(-d^{\nu} t^{\nu}\right)
\end{aligned}
$$

\section{Conclusion}

In this paper, we establish some fractional and integral representations of generalized $\mathrm{k}-$ Bessel function. Also, we give the solution of fractional kinetic equation involving k-Bessel function with the help of Sumudu transform. This paper conclude with the remark that, the results given in this paper are general and can lead to yield many fractional integrals (derivatives) involving the Bessel, generalized Bessel and trigonometric functions by the suitable specializations of arbitrary parameters in the theorems and corollaries. 


\section{REFERENCES}

[1] M.A. Asiru, Sumudu transform and the solution of integral equation of convolution type. International Journal of Mathematical Education in Science and Technology. 32, 906-910(2001)

[2] F.B.M. Belgacem, A.A. Karaballi, S.L. Kalla, Analytical Investigations of the Sumudu Transform and Applications to Integral Production Equations. Journal of Mathematical Problems in Engineering. 3, 103-118(2003)

[3] F.B.M. Belgacem, A.A. Karaballi, Sumudu transform fundamental properties investigations and applications. International J. Appl. Math. Stoch. Anal. (2005) 1-23.

[4] F.B.M. Belgacem, A.A. Karaballi, Sumudu Transform Fundamental Properties Investigations and Applications. Journal of Applied Mathematics and Stochastic Analysis. 2006,123(2006)

[5] F.B.M. Belgacem, Introducing and Analyzing Deeper Sumudu Properties. Nonlinear Studies. 13, 23-42(2006)

[6] F.B.M. Belgacem, Applications with the Sumudu Transform to Bessel Functions and Equations. Applied Math. Sciences. 4, 3665-3686(2010)

[7] F.B.M. Belgacem, Sumudu applications to Maxwells equations. PIERS Online, 5, 355-360(2009)

[8] F.B.M. Belgacem, E.H. Al-Shemas, R. Silambarasan, Sumudu computation of the transient magnetic field in a lossy medium. Applied Mathematics \& Information Sciences. 6, 1-9(2016)

[9] F.B.M. Belgacem, R. Silambarasan, A distinctive Sumudu treatment of trigonometric functions. Journal of Computational and Applied Mathematics. 312, 74-81(2017)

[10] R.A. Cerutti, On the k-Bessel Functions, International Mathematical Forum, Vol. 7, 2012, no. 38, 1851 - 1857

[11] A. Chouhan, S.D. Purohit, and S. Saraswat, An alternative method for solving generalized differential equations of fractional order, Kragujevac J. Math., 37(2) (2013), 299-306.

[12] V.B.L. Chaurasia and S.C. Pandey, On the new computable solution of the generalized fractional kinetic equations involving the generalized function for the fractional calculus and related functions, Astrophys. Space Sci., 317 (2008), 213-219.

[13] R. Diaz and E. Pariguan, On hypergeometric functions and k-Pochhammer symbol, Divulgaciones Matematicas Vol.15, 2 (2007), 179-192

[14] A. Erdélyi, W. Magnus, F. Oberhettinger, and F.G. Tricomi, Tables of Integral Transforms, Vol. 1, McGraw-Hill, New York-Toronto-London, 1954.

[15] C. Fox, The asymptotic expansion of generalized hypergeometric functions, Proc. London. Math. Soc., 27(4) (1928), 389-400.

[16] K.S. Gehlot, S.D. Purohit Fractional calculus of K-Bessel's function, Acta Universitatis Apulensis, No. 38/2014 pp. 273-278

[17] V.G. Gupta, B. Sharma and F. B. M. Belgacem, On the solutions of generalized fractional kinetic equations, Applied Mathematical Sciences, 5(19) (2011), 899-910.

[18] A. Gupta and C.L. Parihar, On solutions of generalized kinetic equations of fractional order, Bol. Soc. Paran. Mat., 32(1) (2014), 181-189.

[19] H.J. Haubold and A.M. Mathai, The fractional kinetic equation and thermonuclear functions, Astrophys. Space Sci., 327 (2000), 53-63.

[20] A.A. Kilbas, N. Sebastian, Generalized fractional integration of Bessel function of the first kind. Int Transf Spec Funct 19(2008), 869-883.

[21] A. A. Kilbas, M. Saigo and J. J. Trujillo, On the generalized Wright function, Fract. Calc. Appl. Anal. 5(4) (2002), $437-460$.

[22] A. A. Kilbas, H. M. Srivastava, and J. J. Trujillo, Theory and Applications of Fractional Differential Equations, North-Holland Mathematics Studies 204, Elsevier, Amsterdam, 2006.

[23] G.M. Mittag-Leffler, Sur la representation analytiqie d'une fonction monogene cinquieme note, Acta Math., 29 (1905), 101-181.

[24] S.R Mondal, Representation Formulae and Monotonicity of the Generalized k-Bessel Functions, arXiv:1611.07499 [math.CA], 2016

[25] K.S. Nisar, S.D. Purohit, S.R. Mondal, Generalized fractional kinetic equations involving generalized Struve function of the first kind. Journal of King Saud University Science, 28, 167-171(2016) 
[26] K.S. Nisar, D. Baleanu, M. Alqurasi, Fractional Calculus and application of generalized Struve functions. SpringerPlus, $5,910(2016)$

[27] K.S. Nisar,K.S., F.B.M. Belgacem, Dynamic k-Struve Sumudu solutions for fractional kinetic equations. Advances in Difference Equations 2017:340 (2017).

[28] K. S. Nisar, F.Qi, On solutions of fractional kinetic equations involving the generalized k-Bessel function, Note di Matematica 37, 1-10 (2017).

[29] E. D. Rainville, Special functions, Macmillan, New York, 1960.

[30] L.G. Romero, G.A. Dorrego and R.A. Cerutti, The k-Bessel Function of the First Kind, International Mathematical Forum, Vol. 7, 38(2012), 1859-1864.

[31] A. Saichev and M. Zaslavsky, Fractional kinetic equations: solutions and applications, Chaos, 7(1997), 753-764.

[32] S.G. Samko, A.A. Kilbas, O.I. Marichev, Fractional Integrals and Derivatives, Theory and Applications. Gordon and Breach, New York, 1993.

[33] R.K. Saxena, A.M. Mathai, and H.J. Haubold, On Fractional kinetic equations, Astrophys. Space Sci., 282 (2002), 281-287.

[34] R.K. Saxena, A.M. Mathai, and H.J. Haubold, On generalized fractional kinetic equations, Physica A 344 (2004), 657-664.

[35] R.K. Saxena, A.M. Mathai and H.J. Haubold, Solution of generalized fractional reaction-diffusion equations, Astrophys. Space Sci., 305 (2006), 305-313.

[36] R.K. Saxena and S.L. Kalla, On the solutions of certain fractional kinetic equations, Appl. Math. Comput., 199 (2008), 504-511.

[37] M.R. Spiegel, Theory and Problems of Laplace Transforms, Schaums Outline Series, McGraw-Hill, New York, 1965.

[38] H.M. Srivastava and R.K. Saxena, Operators of fractional integration and their applications, Appl. Math. Comput., 118 (2001), 1-52.

[39] G.K. Watugala, Sumudu transform:a new integral transform to solve differential equations and control engineering problems. Int.J.Math. Edu.Sci.Tech. 24, 35-43(1993)

[40] G.K. Watugala, The Sumudu transform for functions of two variables, Math. Eng.Ind. 8, 293-302(2002)

[41] A. Wiman, Uber den fundamental satz in der theorie der funktionen $E_{\alpha}(z)$, Acta Math. 29 (1905), 191-201.

[42] E. M. Wright, The asymptotic expansion of integral functions defined by Taylor series, Philos. Trans. Roy. Soc. London, Ser. A. 238 (1940), 423-451.

[43] E. M. Wright, The asymptotic expansion of the generalized hypergeometric function, Proc. London Math. Soc. (2) 46(1940), 389-408.

[44] G.M. Zaslavsky, Fractional kinetic equation for Hamiltonian chaos, Physica D, 76(1994), 110-122.

K. S. Nisar : ${ }^{1}$ Department of Mathematics, College of Arts and Science-Wadi Aldawaser, 11991, Prince Sattam bin Abdulaziz University, Alkharj, Saudi Arabia

E-mail address: ksnisar1@gmail.com, n.sooppy@psau.edu.sa

M.S. Abouzaid: ${ }^{2}$ Department of Mathematics, College of Arts and Science-Wadi Aldawaser, 11991, Prince Sattam bin Abdulaziz University, Alkharj, Saudi Arabia.

${ }^{3}$ Department of Mathematics, Faculty of Science, Kafrelshiekh University, Egypt

E-mail address: moheb_abouzaid@hotmail.com

F.B.M. Belgacem: ${ }^{4}$ Department of Mathematics, Faculty of Basic Education, PaAet, Al-Ardhiya, Kuwait. E-mail address: fbmbelgacem@gmail.com 